\title{
Development of a Poly( $N$-vinyl-2-pyrrolidone)/Poly (ethylene glycol) Hydrogel Membrane Reinforced with Methyl Methacrylate-Grafted Polypropylene Fibers for Possible Use as Wound Dressing
}

\author{
L. C. Lopérgolo, ${ }^{1,2}$ A. B. Lugão, ${ }^{1}$ L. H. Catalain ${ }^{2}$ \\ ${ }^{1}$ Instituto de Pesquisas Energéticas e Nucleares IPEN-CNEN/SP - Travessa R, 400, 05508-970, São Paulo-SP, Brazil \\ ${ }^{2}$ Instituto de Química, Universidade de São Paulo, CP 26.077, 05513-970 São Paulo-SP, Brazil
}

Received 10 April 2001; accepted 14 January 2002

Published online 00 Month 2002 in Wiley InterScience (www.interscience.wiley.com). DOI: 10.1002/app.10929

\begin{abstract}
A hydrogel is a polymeric material that exhibits the ability to swell in water and retains a significant fraction of water within its structure, but does not dissolve in water. One of the major problems in the application of these materials is their relatively poor mechanical strength, attributed to the high degree of hydration of the gel. This work was directed to the study of the interactions between hydrophobic and hydrophilized fibers, with the objective of optimization of the mechanical properties of poly $(\mathrm{N}$-vinyl-2-pyrrolidone) membranes. The membranes were prepared by electron-beam irradiation of an aqueous polymer solution. A nonwoven cloth made of polypro-
\end{abstract}

pylene matted fiber, grafted with methyl methacrylate, was employed as a reinforcement. The changes in the main properties of the membranes, such as the gel content, swelling characteristics, cytotoxicity, and mechanical behavior, were investigated. The results showed an increase of $800 \%$ in tensile strength, without changes in the swelling and cytotoxicity. () 2002 Wiley Periodicals, Inc. J Appl Polym Sci 86: 000-000, 2002

Key words: hydrogels; mechanical properties; radiation; biomaterials; membranes

\section{INTRODUCTION}

A hydrogel is defined as a polymeric material that exhibits the ability to swell in water and retain a significant fraction of water within its structure, but does not dissolve in water. Since the development of 2-hydroxyethyl methacrylate (HEMA) gels in early $1960 \mathrm{~s}^{1}$ and the discovery in 1962 that epithelialization can be accelerated if the wound is kept moist by using an occlusive polyethylene film, ${ }^{2}$ hydrophilic gels have frequently been used as biocompatible interfaces in a variety of applications and a continuous experimental development of occlusive films has occurred.

The third generation of occlusive wound dressings appeared in the early $1990 \mathrm{~s}^{3}$; these hydrogels are transparent, water absorbent (up to $96 \%$ of their weight ${ }^{4,5}$ ), and nonadherent. Hydrogels are more often defined as two-component systems, where the first is a hydrophilic polymer, insoluble in water due the existing three-dimensional network joining its chains,

Correspondence to: L. H. Catalani (catalani@iq.usp.br).

Contract grant sponsor: Fundação de Amparo a Pesquisa do Estado de São Paulo, FAPESP, São Paulo, Brazil.

Journal of Applied Polymer Science, Vol. 86, 000-000 (2002) (C) 2002 Wiley Periodicals, Inc. and the second one is water. ${ }^{6}$ These systems may swell in water up to a certain equilibrium state and retain their original shape. The main areas of hydrogel applications today include (i) topical applications as wound dressings; (ii) drug-delivery systems; (iii) transdermal systems; (iv) dental applications; (v) injectable polymers; (vi) implants; (vii) ophthalmic applications; and (viii) stimuli-responsive systems. ${ }^{7}$

Hydrogel dressings were originally invented as wound burn dressings. Since the beginning of their commercialization, sold under the trade name $\mathrm{HDR}^{\circledR}$ and AQUA-GEL ${ }^{\circledR}$, they have also been used for medical treatment of other types of wounds and illnesses. The membranes developed by Rosiak consists of water, poly(N-vinyl-2-pyrrolidone) (PVP), poly(ethylene glycol) (PEG), and agar, the last used as a premolding. In the end-use form, they are supplied as transparent sheets with a thickness of a few millimeters, containing over $90 \%$ of water. ${ }^{8}$ One of the major problems in the application of these materials is their relatively poor mechanical strength. Other polymeric materials used in association with PVP include $\kappa$-carrageenan, ${ }^{9}$ chitosan, ${ }^{10}$ and poly(ethylene oxide). ${ }^{11}$

Wound dressing produced by the Rosiak method ${ }^{8}$ (see Experimental section) is now being introduced into the Brazilian market. However, such hydrogel dressings usually require extreme care in handling to 
avoid breakage or deformation. This lack of good mechanical properties is attributed to the high degree of hydration of the membrane. ${ }^{12}$ Therefore, strengthening of the composite is a primary requirement for its proper market acceptance.

Due to the hydrophilic nature of the polymeric chains, they lose mechanical properties as they absorb water. ${ }^{13,14}$ Hilmy et al. ${ }^{4}$ studied the mechanical properties, degree of swelling, and resistance to bacterial migration of a hydrogel composed of PVP, agar, and PEG. They observed that the addition of agar and PEG improved the mechanical properties of the PVP membrane.

The reinforcement of its polymeric structure with hydrophobic fibers is a usual attempt to overcome this drawback. An U.S. patent ${ }^{15}$ describes the use of a PE film placed on top of the gel. Another patent ${ }^{16}$ describes a dressing composed by a net of defined holes encapsulated by the gel. These solutions, however, present disadvantages, such as preclusion of fast gas flow $\left(\mathrm{O}_{2}\right.$ and $\left.\mathrm{CO}_{2}\right)$ or loss of exudate fluids.

In this work, polypropylene (PP), nonwoven, grafted with methyl methacrylate (MMA), was studied as a reinforcement for the improvement of the mechanical properties of PVP membranes when prepared by electron-beam irradiation. The effects of the gel content, swelling capacity, mechanical properties, and cytotoxicity analysis of the membranes were investigated.

\section{EXPERIMENTAL}

\section{Materials}

Poly(N-vinyl-2-pyrrolidone) (NVP), known as Plasdone K-90 $\left(M_{w}=1.2 \times 10^{6}\right)$, was from GAF Chemical Co. (USA); PEG (ATPEG 600), from OXITENO (Brazil); agar, from OXOID (Brazil); and isotatic polypropylene, donated by FITESA (Brazil). Polypropylene is presented as a cloth made of nonwoven matted fibers.

\section{Preparation of PVP hydrogel ${ }^{8}$}

PVP membranes were prepared by irradiation of an aqueous solution of a polymer sealed in a polyethylene mold. The composition of the homogeneous aqueous solution, which contained 8\% wt PVP with 1.5\% wt PEG and $0.5 \%$ wt agar, was chosen to give a gel as close as possible to commercial dressings. This solution was poured into the mold and covered with a thin polyethylene sheet. The mold was subjected to an electron beam, type "Dynamitron" of Radiation Dynamics, with a maximum energy of $1.5 \mathrm{MeV}$ and 15 $\mathrm{mA}$. The radiation dose was $25 \mathrm{kGy}$, at a dose rate of $11.3 \mathrm{kGy} / \mathrm{s}$ and $25^{\circ} \mathrm{C}$.

\section{Grafting PP fibers with MMA}

The grafted fibers were prepared by a direct method, as described in Lopérgolo et al. ${ }^{17}$ : The fibers were immersed in a methanol/decaline $(3: 2 \mathrm{v} / \mathrm{v})$ solution containing $0.05 \% \mathrm{FeCl}_{3}$ and the acrylic monomer in concentrations varying from 5 to $59 \%$ (w/w), deaerated by $\mathrm{N}_{2}$ bubbling for $10 \mathrm{~min}$, and submitted to electron-beam irradiation with a dose of $20 \mathrm{kGy}$ (dose rate of $11.3 \mathrm{kGy} / \mathrm{s}$, at $25^{\circ} \mathrm{C}$ ).

\section{Preparation of reinforced PVP hydrogel}

When reinforcement was used, the arrangement adopted was of the "sandwich" type, with the PP fibers in the middle layer and the PVP solution on top and bottom of the mold. Three types of the membranes were prepared: (i) PVP membranes without reinforcement; (ii) reinforced PVP membranes with ungrafted PP fibers; and (iii) reinforced PVP membranes with grafted PP fibers $(50,80$, and $150 \%$ wt of MMA).

\section{Gel content of PVP aqueous solution after irradiation}

PVP membranes were subjected to Soxhlet extraction with water for $36 \mathrm{~h}$ to remove the sol content and then dried under a vacuum at $60^{\circ} \mathrm{C}$ for $48 \mathrm{~h}$ to a constant weight, according to ASTM-D3616-88. For the calculation of the gel content of the reinforced membranes, the PP mass was subtracted. The gel content was calculated as follows:

$$
S(\%)=\left(\frac{W_{g}-W_{0}}{W_{g}}\right) \times 100 \quad G(\%)=100-S
$$

where $S(\%)$ is the sol content; $G(\%)$, the gel content; $W_{g}$, the weight of PVP used to prepare the hydrogel before irradiation; and $W_{0}$, the weight of the dried gel.

\section{Swelling capacity}

PVP membranes were immersed in distilled water at ambient temperature until equilibrated. Then, they were removed from the water, dried quickly with filter paper to remove excess water, and weighed. The swelling capacity, in percent (SC \%), was calculated as follows:

$$
S C(\%)=\left(\frac{W_{s}-W_{i}}{W_{i}}\right) \times 100
$$

where $W_{s}$ is the weight of the swollen hydrogel, and $W_{i}$, the initial weight of the hydrogel. 
\begin{tabular}{|l|l|l|l|l|l|l|}
\hline tapraid5/8e-polyapp/8e-polyapp/8e-orig/8e7229d02a & heckt & $\mathrm{S}=8$ & $5 / 3 / 02$ & $12: 35$ & Art: 10577 & Input-DCT-srh \\
\hline
\end{tabular}

NVP/PEG HYDROGEL MEMBRANE FOR WOUND DRESSING

\section{Cytotoxicity tests}

The cytotoxicity of the membranes was tested by an in vitro cell viability method according to Ciapetti et al. ${ }^{18}$ The test was carried out with dilution of the extracts of hydrogel membranes, in contact with a mouse subconjuntive tissue cell culture, from NCTC Clone 929 line (ATCC-CCL1). The phenol solution (0.02\%) and poly(vinyl chloride) (PVC) extracts were used as positive and negative controls, respectively.

\section{Mechanical analysis}

Mechanical properties, such as elongation at break $\left(E_{b}\right)$ and tensile strength $\left(T_{s}\right)$, were determined using an INSTRON Model 5567 tensile testing apparatus at a crosshead speed of $25 \mathrm{~mm} / \mathrm{min}$. The samples were prepared according to ABNT/NBR/6241/80 type I. The measurements were performed at ambient temperature.

\section{RESULTS AND DISCUSSION}

The formation of hydrogels using high-energy radiation can be simply explained as the result of the reciprocal recombination of macroradicals. These, in turn, are generated as a consequence of the direct interaction of the radiation with the polymer or with radicals generated during water radiolysis. If radicals located on different polymer chains are favorably positioned, their recombination results in covalent bonds between polymer chains. If bond-formation processes are faster than are concurrent degradation reactions, then an insoluble gel fraction is formed. Continuous irradiation of such a system increases the amount of the gel, although a part of the macromolecules may still be left unbound (sol). ${ }^{19}$

Gel content measurement is an effective method for evaluating the degree of crosslinking of the material. The optimal conditions for PVP hydrogel formation were previously determined. ${ }^{20}$ The typical hydrogel thus produced presents $85-90 \%$ of gel content, ${ }^{21}$ as a result of the high efficiency of the formation and recombination of polymeric macroradicals with respect to other competitive reactions.

In a previous work, the preparation methodology and characterization of PP fibers grafted with MMA were reported. ${ }^{17}$ That study showed that increasing amounts of MMA grafted onto a cloth of nonwoven PP matted fibers resulted in a severe decrease of their mechanical properties. On the other hand, water compatibility increases, as shown by the swelling measurements. If this product is to provide reinforcement of our membranes, it has to show (i) enhancement of the mechanical properties of the hydrogel, together with (ii) low interference with the hydrogel produc-
TABLE I

Swelling Capacity at $25^{\circ} \mathrm{C}$

\begin{tabular}{cccr}
\hline \multirow{2}{*}{$\begin{array}{c}\text { Time of } \\
\text { swelling }(\mathrm{h})\end{array}$} & \multicolumn{3}{c}{$(\%)$ Swelling } \\
\cline { 2 - 4 } & $\mathrm{A}$ & $\mathrm{B}$ & $\mathrm{C}$ \\
\hline 0.5 & $48 \pm 9$ & $30 \pm 12$ & $42 \pm 4$ \\
1 & $60 \pm 8$ & $45 \pm 11$ & $50 \pm 7$ \\
2 & $115 \pm 14$ & $87 \pm 20$ & $105 \pm 7$ \\
3 & $145 \pm 8$ & $128 \pm 12$ & $136 \pm 6$ \\
4 & $180 \pm 8$ & $163 \pm 12$ & $173 \pm 5$ \\
5 & $225 \pm 11$ & $205 \pm 14$ & $223 \pm 2$ \\
12 & $385 \pm 11$ & $362 \pm 16$ & $375 \pm 7$ \\
24 & $380 \pm 8$ & $365 \pm 11$ & $376 \pm 3$ \\
72 & $385 \pm 7$ & $355 \pm 15$ & $368 \pm 3$ \\
\hline
\end{tabular}

A, membrane without reinforcement; B, reinforced membrane using nongrafted PP fibers; C, reinforced membrane with 50\% MMA grafted PP fibers.

tion process and its main properties, which are (iii) good swelling characteristics and (iv) lack of cytotoxicity.

The gel contents were calculated for membranes from an $8 \%$ PVP solution in water prepared (i) without any reinforcement, (ii) with PP fibers (nonwoven cloth) as a mechanical reinforcement, and (iii) with PP fibers grafted with MMA as the reinforcement. The numbers found were $88.6 \pm 2.3 \%, 87.9 \pm 2.7 \%$, and $88.9 \pm 3.1 \%$, respectively. These results show that the use of reinforcement does not interfere with the degree of crosslinking and gel formation.

The equilibrium swelling of the membranes, measured at $25^{\circ} \mathrm{C}$, was reached after $12 \mathrm{~h}$. Table I shows T1 the swelling capacity of the basic membrane, compared with a PP fiber-reinforced membrane and a membrane with grafted PP fiber reinforcement $(50 \%$ of MMA). As can be observed, the swelling capacity did not undergo significant alteration. Again, this result is important to show that, in spite of the presence of a very hydrophobic component, the water absorbance capacity of the original membrane was not altered.

The alterations at the molecular level caused by radiation and grafting could provoke changes of macroscopic properties. One of these important mechanical properties is the tensile strength. As shown before, ${ }^{17}$ the tensile strength decreases with an increasing degree of grafting in PP fibers.

This behavior is different when grafted PP fibers are introduced as reinforcement in PVP membranes. As can be seen in Figure 1, the tensile strength F1 increased 3.5-fold when the original (nongrafted) PP fibers were used as reinforcement. With grafted reinforcement, the tensile strength increased with an increasing degree of grafting, leveling off at about $80 \%$ grafting. This corresponds to a gain of $800 \%$ (0.02-0.16M Pa) over the initial value. These results are excellent when compared with other approach- 
$\mathrm{es}^{22,23}$ for enhancing the mechanical strength of PVP membranes, where a maximum of $20 \%$ improvement was reached.

Figure 1 also shows the elongation at break in reinforced membranes. As can be observed, the elongation decreased substantially when PP fibers were used. This is somewhat expected, taking into account the lower plasticity of PP as compared to the membrane, imposing the original PP elongation to the reinforced membrane.

In both cases (tensile strength and elongation at break), the grafted and nongrafted reinforced membranes show different behavior, pointing to strong chemical interaction between the PVP membrane and the grafted PP fibers. As a compromise between the increase in tensile strength with the decrease of elongation, a $50-80 \%$ MMA grafting would appear to be ideal.

The cytotoxicity of the modified membranes was tested according to Ciapetti et al. ${ }^{18}$ In Figure 2, the relative count of total visible cells is plotted against the membrane extract concentration for a PVP membrane reinforced with 50\% MMA-grafted PP fibers. For comparison, phenol was used as a positive control, and PVC, as a negative control. The conclusion from this experiment is that PP grafted by MMA does not impose any toxic effect on the user.

\section{CONCLUSIONS}

The use of PP fibers as a coadjuvant in the production of PVP membranes provides the best alternative method for substantially improving their mechanical properties, with a gain of up to eightfold over the

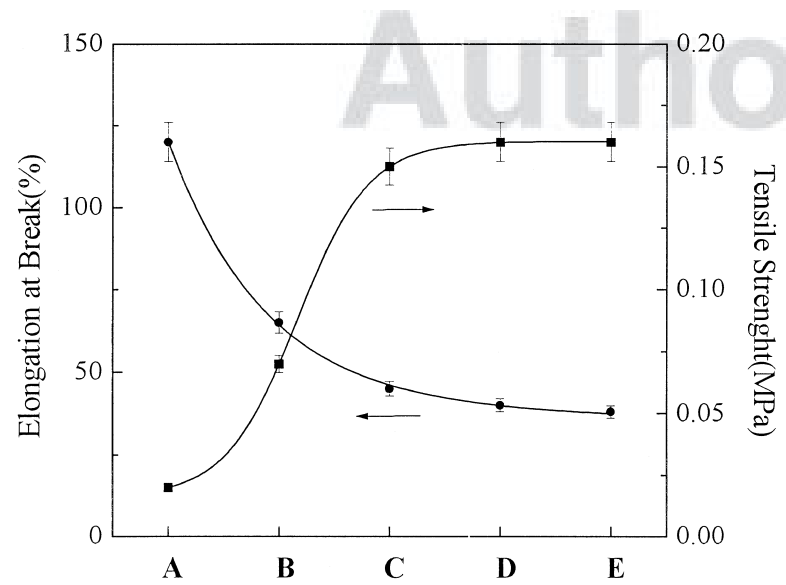

Figure 1 Effect of reinforcement on $(\mathbf{\square})$ tensile strength $\left(T_{s}\right)$ and $(\mathbf{O})$ elongation at break $\left(E_{b}\right)$. (A) Membrane without reinforcement; (B) reinforced membrane using nongrafted PP fibers; (C) reinforced membrane with 50\% MMA-grafted PP fibers; (D) reinforced membrane with $80 \%$ MMA-grafted PP fibers; (E) reinforced membrane with 150\% MMA-grafted PP fibers.

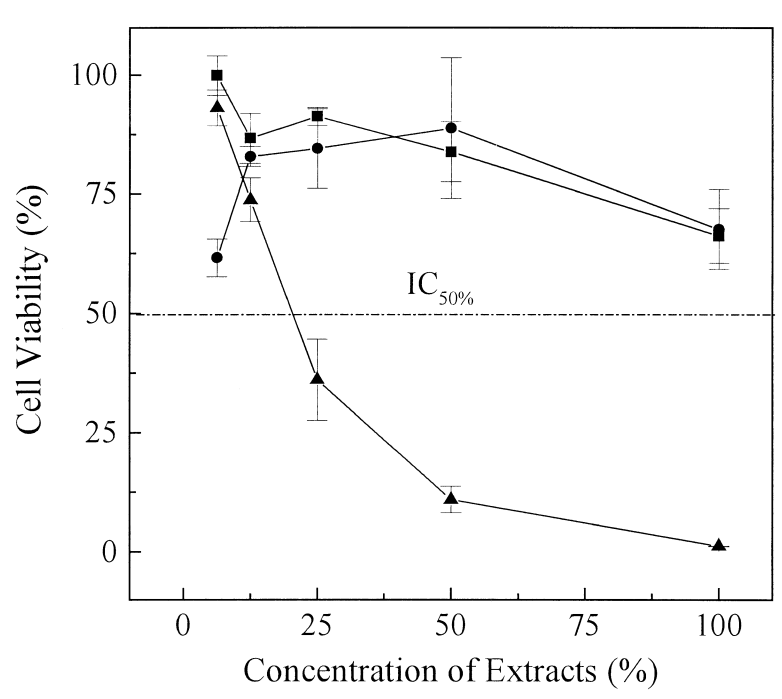

Figure 2 Colony suppression curves of the hydrogel membranes: (- - ) PVP membrane with PP reinforcement grafted with 50\% MMA; (- - negative control (PVC); (一 $\square$-) positive control (phenol).

original tensile strength. The grafting of these PP fibers with MMA, which improves their hydrophilicity, results in higher compatibility with the aqueous medium of the PVP membrane and, hence, a better product without limiting the important characteristics of the membrane.

The authors would like thank Dr. Sizue O. Rogero and the Instituto Adolfo Lutz for their contribution to the cytotoxicity tests, Prof. Frank H. Quina (Universidade de São Paulo) for kindly reviewing this manuscript, and the Fundação de Amparo a Pesquisa do Estado de São Paulo, FAPESP, São Paulo, Brazil, for financial support.

\section{References}

1. Wichterle, O.; Lim, D. Nature 1960, 185, 117-118.

2. Winter, A. Nature 1962, 45, 89-90.

3. Rosiak, J. M.; Olejniczak, J. Radiat Phys Chem 1993, 42(4-6) 903-906.

4. Hilmy, N.; Darwis, D.; Hardiningsih, L. Radiat Phys Chem 1993, 42(4-6), 911- 914 .

5. Ichijo, H.; Hirasa, O.; Kishi, R.; Oowada, M.; Sahara, K.; Kokufuta, E.; Kohno, S. Radiat Phys Chem 1995, 46, 185-190.

6. Rosiak, J.; Olejniczak, J.; Charlesby, A. Radiat Phys Chem 1988, 32, 5, 691-694.

7. Rosiak, J. M. J Control Rel 1994, 31, 9-19.

8. Rosiak, J. M.; et al. U.S. Patent 4871 490, 1989.

9. Maolin, Z.; Hongfei, H.; Yosshii, F.; Makuuchi, K. Radiat Phys Chem 2000, 57, 459-464.

10. Risbud, M.; Hardikar, A.; Bhat, S.; Bhonde, R. J Control Rel 2000, 68, 23-30.

11. Alvarez, M.; Lugão, A.; Miranda, A.; Nakashira, H.; Miranda, L.; Souza, A.; Moreira, E.; Oliveira, G.; Andrade e Silva, L. III Encontro Nacional de Biomateriais, Anais, São Paulo-Brazil, 1996; p 37. 
12. Rosiak, J. M. In Radiation Effects on Polymers; Clough, R. C.; Shalab, S. W., Eds.; American Chemical Society: Washington, DC, 1991.

13. Rosiak, J. M.; Ulanski, P.; Pajewski, L. A.; Yoshii, F.; Makuuchi, K. Radiat Phys Chem 1995, 46, 2, 161-168.

14. Lugão, A. B.; Machado, L. D. B.; Miranda, L. F.; Alvarez, M. R.; Rosiak, J. M. Radiat Phys Chem 1998, 52(1-6), 319-322.

15. Schonfeld, E.; McGinity, J. U.S. Patent 4646 730, 1987.

16. Fabo, T. U.S. Patent 4921 704, 1990.

17. Lopérgolo, L. C.; Catalani, L. H.; Machado, L. D. B.; Rela, P. R.; Lugão, A. B. Radiat Phys Chem 2000, 57(3-6), 451-454.
18. Ciapetti, G.; Granchi, D.; Verri, E.; Savarino, L.; Cavedagna, D.; Pizzoferrato, A. Biomaterials 1996, 17, 1259-1264.

19. Makuuchi, K. Hydrogel; Takasaki Radiation Chemistry Research Establishment, JAERI: 1994; pp 1-8.

20. Miranda, L. F.; Lugão, A. B.; Machado, L. D. B.; Ramanathan, L. V. Radiat Phys Chem 1999, 55, 709-712.

21. Sem, M.; Guven, O. Radiat Phys Chem 1999, 55, 113-120.

22. Higa, O. Z.; Rogero, S. O.; Machado, L. D. B.; Mathor, M. B.; Lugão A. B. Radiat Phys Chem 1999, 55, 705-707.

23. Baccaro, S.; Pajewski, L. A.; Scoccia, G.; Volpe, R.; Rosiak, J. M. Nucl Instrum Meth B 1995, 105(1-4), 100-102. 